\title{
Inequalities for a Matrix Riccati Equation*
}

\author{
RAYMOND REDHEFFER
}

Communicated by E. HILLE

1. Introduction. Let $A, B, C$ and $D$ be complex $n \times n$ matrix functions of the real variable $y$, and let $U, R, S, W$ be complex $n \times n$ matrix functions of $(x, y)$. Generalizing a scalar system introduced by the author, W. T. REID [1] studies the matrix system

$$
\begin{aligned}
U_{\nu} & =A+D U+U B+U C U, & U(x, x) & =0, \\
R_{\nu} & =R(B+C U), & R(x, x) & =1, \\
S_{\nu} & =(D+U C) S, & S(x, x) & =1, \\
W_{\nu} & =R C S, & W(x, x) & =0,
\end{aligned}
$$

where 1 denotes the identity matrix. As in [2] no special restriction on the coefficients is assumed but the region in which (1) holds has a property analogous to convexity.

When $n=1$ the system (1) corresponds to the nonbilateral case in the physical interpretation of [2]-[4]. The dielectric medium can be chosen as anisotropic, so that the transmission coefficients $S$ and $R$ in opposite directions need not be equal. Since the change of variable $T=(R S)^{\frac{1}{3}}$ reduces the nonbilateral to the bilateral case, this alteration is of small moment when $n=1$; but it is an important increase in generality when $n>1$.

In the scalar case a simple change of variable reduces (1) to a linear system, which can be written in matrix form; see [2], Theorem VIII. It is observed by REID that the analogous change of variable (constructed with due regard to the noncommutativity of matrix multiplication) also linearizes the system for $n>1$. By its aid he is able to prove uniqueness of the solution very simply - a possibility which eluded the present author, despite the fact that the linear system was available to him. (The usual Lipschitz argument does not apply, since we have no hypothesis on the coefficients.)

* The preparation of this work was sponsored in part by the Office of Ordnance Research, U. S. Army. Reproduction in whole or in part is permitted for any purpose of the United States Government. 
In [3] it was shown that the functional equations associated with the Riccati system follow at once from uniqueness. Indeed, these functional equations were found to be just the semi-group property, the "major premise of Huygen's principle" according to HADAMARD. One purpose of the present note is to study the extension of this result to REID's system (1). In view of REID's uniqueness theorem, we are able to give a unified derivation of the four functional equations, which is brief and also makes their meaning clear. The author was surprised to find that the equations are not quite equivalent to the closure property; they say something more.

Another purpose is to sharpen the inequalities given in [1]. According to REID the matrix

$$
U(x, y, K)=U+S K(1-W K)^{-1} R
$$

satisfies the first equation (1) and, as is evident,

$$
U(x, x, K)=K \text {. }
$$

(Although REID uses the linear system, the result can also be verified by direct substitution in (1) as in [3], Theorem I. Since $\left(A^{-1}\right)_{y}=-A^{-1} A_{y} A^{-1}$ the inverse in (2) causes no trouble, and it will be found that the noncommutativity also causes no trouble.)

We shall denote the length of a complex vector $V$ by $|V|$ and the norm of a matrix $A$ by

$$
\|A\|=\sup |A V| \text { for }|V|=1 \text {. }
$$

The matrices $U, R, S, W$ will be said to have Property $\mathrm{P}$ if, in (2),

$$
\|U(x, y, K)\|<1 \text { whenever }\|K\|<1 .
$$

In ReID's notation $U(x, y, K)$ has Property $\odot$ if it has Property $\mathrm{P}$ for all $x \varepsilon I$, $y \varepsilon I, y \geqq x$, where $I$ is a given interval such that (1) holds in $I \times I$. When $A, B, C, D$ are piecewise continuous he shows that the condition

$$
\left[\begin{array}{cc}
D^{*}+D & A+C^{*} \\
A^{*}+C & B+B^{*}
\end{array}\right] \leqq 0, \quad y \varepsilon I,
$$

is sufficient for Property $\odot$, and that it is both necessary and sufficient when $D=B^{\prime}, A=A^{\prime}, C=C^{\prime}$. Since the latter conditions hold automatically in the bilateral case for $n=1$, REID says that his theorem generalizes Theorem IV of [3]. However, the conclusion of Theorem IV is not only that Property $\odot$ holds, but that

$$
\left\|\left[\begin{array}{cc}
S & U \\
W & R
\end{array}\right]\right\| \leqq 1 \text { for } x \varepsilon I, \quad y \varepsilon I, \quad y \geqq x .
$$

Equation (6) is easily shown to imply Property $P$, but the converse is false even for $n=1$. Thus REID's Theorem 4.2 is more accurately a generalization of [3], 
Theorem III than [3], Theorem IV. We shall obtain the desired generalization of IV, showing that (5) is a necessary and sufficient condition for (6) without regard to the symmetry of the matrices.

REID remarks that he does not know a necessary and sufficient condition for Property $\odot$ in the general case. However, use of REID's result in the bilateral case yields (5), and, as we have noted, this in turn yields (6). Thus Property $\mathrm{P}$ is completely equivalent to (6) in that case. This suggests the purely algebraical result that if

$$
\left\|U+S K(1-W K)^{-1} R\right\|<1 \text { for }\|K\|<1,
$$

if $U=U^{\prime}, R=S^{\prime}, W=W^{\prime}$, and if $R$ is nonsingular, then

$$
\left\|\left[\begin{array}{cc}
S & U \\
W & R
\end{array}\right]\right\| \leqq 1 .
$$

In Theorem IX we give a direct proof of the equivalence of (7) and (8) under the above-mentioned conditions, without reference to Riccati equations. Thus REID's Theorem 4.2 generalizes the author's Theorem IV, after all, provided one can take for granted this algebraical theorem.

Knowing that (8) holds we have also

$$
\left\|W+R K(1-U K)^{-1} S\right\|<1 \text { for }\|K\|<1
$$

as a consequence of (7) and the assumed symmetry. However, (7) and (9) are equivalent under the sole hypothesis that $R S$ is nonsingular; the symmetry is not required. This fact is established in Theorem VI.

In studying the relations between (7), (8), and (9) the author came upon a criterion for property $\mathrm{P}$ which can be used to give a necessary and sufficient condition for Property $\odot$. This condition, which answers the question raised by REID, is presented in Theorem XII.

Some of the results of [3] refer, for $n=1$, to conditions under which

$$
U+S K(1-W K)^{-1} R
$$

is unitary for all unitary $K$. This property, termed Property U, is briefly considered here too, as an aid in checking the other developments. It is found that the conditions for Property $U$ in the purely algebraic setting become unexpectedly restrictive when applied to solutions of (1).

Concluding these introductory remarks, we mention some ramifications of the problems considered here. Many of the facts established about (7)-(9) carry over to Hilbert space; so that one can develop the theory of linear fractional transformations of operators in a way that closely parallels the development for $n=1$. Again in [3] it was shown how an interesting problem in diffusion, considered by MYcielski \& PAszkowski [5], can be generalized and efficiently solved by the study of (1) for $n=1$. In [6] the problems of [5] are extended to $n$ dimensions. When the functional equations of [6] are written in matrix form 
they are seen to be identical with those considered by REID. Thus the generalized diffusion problem of [6] can also be attacked by the methods developed here. Finally, since these questions all have their origin in network analysis [4], it would be expected that REID's generalization would lead to interesting results in that field. This is the case; indeed, the general theory of the scattering matrix as developed by Schwinger, Dicke, Twersky and others can be put, by the present methods, into the algebraic framework of [4].

2. Closure and the functional equations. Suppose the solution $U(x, y, K)$ has the value $K_{1}$ at $\left(x_{0}, y_{1}\right)$, so that

$$
U\left(x_{0}, y, K\right)=K_{1} \quad \text { at } \quad y=y_{1} .
$$

Since $U(x, x, K) \equiv K$ we have also

$$
U\left(y_{1}, y, K_{1}\right)=K_{1} \text { at } y=y_{1} .
$$

By uniqueness these solutions coincide, and hence $U\left(y_{1}, y, K_{1}\right) \equiv U\left(x_{0}, y, K\right)$. Upon replacing $K_{1}$ by its value (10) and renaming the variables we obtain the identity

$$
U(x, z, K) \equiv U[y, z, U(x, y, K)] .
$$

This result, the matrix analogue of Theorem II in [3], is nothing more than the statement of the familiar closure or semigroup property for the system (1).

We shall distinguish variables more briefly by subscripts, writing

$$
U(x, y)=U_{12}, \quad U(x, z)=U_{13}, \quad U(y, z)=U_{23}
$$

and similarly for $R, S, W$. If (11) is combined with (2) we get the identity

$$
\begin{aligned}
U_{13}+S_{13} K\left(1-W_{13} K\right)^{-1} R_{13} & =U_{23}+S_{23}\left[U_{12}+S_{12} K\left(1-W_{12} K\right)^{-1} R_{12}\right] \\
\cdot & \left\{1-W_{23}\left[U_{12}+S_{12} K\left(1-W_{12} K\right)^{-1} R_{12}\right]\right\}^{-1} R_{23}
\end{aligned}
$$

which will now be used to obtain REID's functional equations.

Theorem I. Let $U_{i j}, R_{i j}, S_{i j}, W_{i j}$ be complex $n \times n$ matrices with $R_{i j}$, $S_{i j}$, and $1-U_{12} W_{23}$ nonsingular and let $\epsilon$ be positive. If (12) holds whenever $\|K\|<\epsilon$, then there is a scalar $\lambda$ such that

$$
\begin{aligned}
U_{13} & =U_{23}+S_{23} U_{12}\left(1-W_{23} U_{12}\right)^{-1} R_{23}, \\
R_{13} & =\lambda R_{12}\left(1-W_{23} U_{12}\right)^{-1} R_{23}, \\
S_{13} & =\lambda^{-1} S_{23}\left(1-U_{12} W_{23}\right)^{-1} S_{12}, \\
W_{13} & =W_{12}+R_{12} W_{23}\left(1-U_{12} W_{23}\right)^{-1} S_{12} .
\end{aligned}
$$

Conversely, if there is such a scalar, then (12) holds for all $K$ for which the expressions have a meaning.

To establish this, note that $K=0$ yields (13a), hence shows that $1-W_{23} U_{12}$ 
is nonsingular. We define matrices $a, b, c$ and $z$ by

$$
\begin{aligned}
R_{13} & =R_{12} a\left(1-W_{23} U_{12}\right)^{-1} R_{23}, \\
S_{13} & =S_{23} b\left(1-U_{12} W_{23}\right)^{-1} S_{12}, \\
W_{12}-W_{13} & =R_{12} c\left(1-U_{12} W_{23}\right)^{-1} S_{12}, \\
K & =\left[W_{12}+z\left(1-U_{12} W_{23}\right)^{-1} S_{12}\right]^{-1},
\end{aligned}
$$

so that $z$ has the same degree of arbitrariness as $K$ does.

Subtract $U_{23}$ from both sides of (12) and use (13a) to eliminate $U_{13}$. Next, since

$$
K(1-W K)^{-1} \equiv\left(K^{-1}-W\right)^{-1}
$$

for nonsingular $K$, we have on the left of (12)

$$
K\left(1-W_{13} K\right)^{-1}=\left[W_{12}-W_{13}+z\left(1-U_{12} W_{23}\right)^{-1} S_{12}\right]^{-1}
$$

and we can now eliminate $W_{13}$ by (16). Similarly $S_{13}$ and $R_{13}$ are eliminated by (14) and (15), so that the resulting equation has only subscripts 12 and 23 , not 13.

The expression in curly braces on the right of (12) becomes, by (17) and (18),

$$
\left(1-W_{23} U_{12}\right)\left(1-W_{23} z^{-1} R_{12}\right) \text {. }
$$

Upon taking the inverse, as required by (12), we find that the resulting equation has the right hand factor $\left(1-W_{23} U_{12}\right)^{-1} R_{23}$ and the left hand factor $S_{23}$. Dividing these out yields a more accessible condition equivalent to (12):

$$
U_{12}+b\left(R_{12} c+z\right)^{-1} R_{12} a=\left[U_{12}+\left(1-U_{12} W_{23}\right) z^{-1} R_{12}\right]\left(1-W_{23} z^{-1} R_{12}\right)^{-1} .
$$

If the right hand side is multiplied by $1-W_{23} z^{-1} R_{12}$ we get

$$
b\left(R_{12} c+z\right)^{-1} R_{12} a\left(1-W_{23} z^{-1} R_{12}\right)=z^{-1} R_{12}
$$

or, rewriting,

$$
R_{12} a\left(R_{12}^{-1} z-W_{23}\right)=\left(R_{12} c+z\right) b^{-1} .
$$

The fact that $z$ is variable now gives

$$
-R_{12} a W_{23}=R_{12} c b^{-1}, \quad R_{12} a R_{12}^{-1} z=z b^{-1} .
$$

Equation (17) shows that we may take $z=p 1$, where $p$ is a sufficiently large constant. Hence $R_{12} a R_{12}^{-1}=b^{-1}$. The fact that both matrices commute with the variable matrix $z$ now shows that each is a scalar: $b=\lambda^{-1} 1, a=\lambda 1$ for some scalar $\lambda$. The first relation (19) yields $c=-W_{23}$, so that (13) follows. Conversely, when $a, b$ and $c$ have these values, then (19) holds for all $z$, and hence (12) holds for all $K$.

If ReID's equations (3.1) are solved for those functions which have argument $(x, z)$ it will be found that they are equivalent to

$$
\begin{aligned}
& U(x, z)=U(y, z)+S(y, z) U(x, y)[1-W(y, z) U(x, y)]^{-1} R(y, z), \\
& R(x, z)=R(x, y)[1-W(y, z) U(x, y)]^{-1} R(y, z),
\end{aligned}
$$




$$
\begin{aligned}
& S(x, z)=S(x, y)[1-U(x, y) W(y, z)]^{-1} S(x, y), \\
& \text { (20d) } \quad W(x, z)=W(x, y)+R(x, y) W(y, z)[1-U(x, y) W(y, z)]^{-1} S(x, y) \text {, }
\end{aligned}
$$

provided the matrices in square brackets are nonsingular. This corresponds to the case $\lambda=1$ in (13), whereas if (13) is written in $(x, y, z)$ notation we have $\lambda=\lambda(x, y, z)$. The system (13) yields the product $S R$ uniquely but does not yield $S$ or $R$. (For $n=1$ this reflects familiar properties of nonbilateral circuits; cf. [4]).

Although the functional equations (13) contain the whole of the information that can be got from HADAMARD's closure principle applied to (1a) we shall find that $\lambda=1$ can be deduced from the differential system (1) as a whole. Thus we have a new proof of (20).

Theorem II. If the functions $U_{12}=U(x, y), \cdots$ considered in Theorem I are solutions of (1), then $\lambda \equiv 1$; that is, (20) holds.

For proof we note by $(13 \mathrm{~b})$ that

$$
\lambda(x, y, z)=R(x, z) R(y, z)^{-1}[1-W(y, z) U(x, y)] R(x, y)^{-1} .
$$

An entirely straightforward calculation yields $\lambda_{z}=0$. Since $\lambda(x, y, y)=1$ by the initial conditions, the desired result follows.

3. The star product. Let $Z_{i}$ be complex $n$-dimensional column vectors and let $U_{i}, R_{i}, S_{i}, W_{i}$ be complex $n \times n$ matrices such that

$$
\begin{aligned}
& {\left[\begin{array}{l}
Z_{3} \\
Z_{6}
\end{array}\right]=\left[\begin{array}{cc}
S_{1} & U_{1} \\
W_{1} & R_{1}
\end{array}\right]\left[\begin{array}{l}
Z_{5} \\
Z_{4}
\end{array}\right]} \\
& {\left[\begin{array}{l}
Z_{1} \\
Z_{4}
\end{array}\right]=\left[\begin{array}{cc}
S_{2} & S_{2} \\
W_{2} & R_{2}
\end{array}\right]\left[\begin{array}{l}
Z_{3} \\
Z_{2}
\end{array}\right] .}
\end{aligned}
$$

This system of $4 n$ equations in $6 n$ unknowns, in general, enables us to find $Z_{1}$ and $Z_{6}$ in terms of $Z_{5}$ and $Z_{2}$, so that

$$
\left(\begin{array}{l}
Z_{1} \\
Z_{6}
\end{array}\right)=\left[\begin{array}{cc}
S_{3} & U_{3} \\
W_{3} & R_{3}
\end{array}\right]\left[\begin{array}{l}
Z_{5} \\
Z_{2}
\end{array}\right] .
$$

By a straightforward calculation one finds that the latter matrix is well determined when $1-U_{12} W_{23}$ and $1-W_{23} U_{12}$ are nonsingular, and that its entries are given by relations having a structure identical with (13) for $\lambda=1$. We shall call this matrix the star product of the preceding matrices; specifically,

$$
\begin{aligned}
& {\left[\begin{array}{cc}
S_{1} & U_{1} \\
W_{1} & R_{1}
\end{array}\right] *\left[\begin{array}{cc}
S_{2} & U_{2} \\
W_{2} & R_{2}
\end{array}\right]} \\
& \quad=\left[\begin{array}{cc}
S_{2}\left(1-U_{1} W_{2}\right)^{-1} S_{1} & U_{2}+S_{2} U_{1}\left(1-W_{2} U_{1}\right)^{-1} R_{2} \\
W_{1}+R_{1} W_{2}\left(1-U_{1} W_{2}\right)^{-1} S_{1} & R_{1}\left(1-W_{2} U_{1}\right)^{-1} R_{2}
\end{array}\right] .
\end{aligned}
$$


Equivalent forms are obtained by use of the identity

$$
A(1-B A)^{-1} \equiv(1-A B)^{-1} A
$$

which holds when the two inverses are meaningful.

The relevance of this operation in the study of Riccati equations stems partly from the following lemma, which is verified by inspection:

Lemma 1. The functional equations (20) associated with (1) are equivalent to the single matrix equation

$$
\left[\begin{array}{ll}
S(x, z) & U(x, z) \\
W(x, z) & R(x, z)
\end{array}\right]=\left[\begin{array}{cc}
S(x, y) & U(x, y) \\
W(x, y) & R(x, y)
\end{array}\right] *\left[\begin{array}{cc}
S(y, z) & U(y, z) \\
W(y, z) & R(y, z)
\end{array}\right] .
$$

Hence this multiplication plays the same role for (1) as does matrix multiplication for the associated linear system. Indeed, a tedious but straightforward calculation shows that the correspondence

$$
\begin{aligned}
{\left[\begin{array}{cc}
S & U \\
W & R
\end{array}\right] \leftrightarrow\left[\begin{array}{cc}
R-W S^{-1} U & W S^{-1} \\
-S^{-1} U & S^{-1}
\end{array}\right] } & \equiv\left[\begin{array}{ll}
R & 0 \\
0 & R
\end{array}\right]+\frac{1}{2}\left[\begin{array}{cc}
W & W \\
1 & 1
\end{array}\right]\left[\begin{array}{cc}
S^{-1} & S^{-1} \\
0 & 0
\end{array}\right]\left[\begin{array}{cc}
-U & 1 \\
-U & 1
\end{array}\right]
\end{aligned}
$$

is an isomorphism. That is, the star product of two such expressions on the left corresponds, under this correspondence, to the matrix product of the two expressions on the right. (For $n=1$ the isomorphism was pointed out in [4].) As a consequence we have

Lemma 2. Star multiplication is associative provided the relevant expressions are well defined.

This fact can be deduced at once from the defining relations (21), without introduction of matrices. However, the isomorphism shows the connection with REID's methods more clearly.

Following [3] we shall say that a $2 n \times 2 n$ matrix is dissipative if its norm is $\leqq 1$. The key lemma, which would tend to justify introduction of the star product, is the following:

Lemma 3. If two matrices are dissipative (or unitary, as the case may be) so is their star product (provided the latter is well defined.)

The proof, given in full in [3], follows by inspection of (21).

The following lemma will be useful to ensure that matrices like $1-U W$ or $1-K W$ for $\|K\| \leqq 1$, which arise throughout our discussion, are nonsingular:

Lemma 4. Let the matrix

$$
\left[\begin{array}{ll}
S & U \\
W & R
\end{array}\right]
$$

be dissipative. If either $R$ or $S$ is nonsingular, then $\|U\|<1$ and $\|W\|<1$. 
The proof is left to the reader. Because of Lemma 4, the annoying proviso in Lemmas 2 and 3 will play no role in the sequel.

4. The main inequality. By Lemmas 1 and 3 we can pass from relations in the small to those in the large in our study of (1). Thus when $y-x=\Delta y \geqq 0$, Equation (1) gives

$$
\left[\begin{array}{cc}
S & U \\
W & R
\end{array}\right]=\left[\begin{array}{ll}
1 & 0 \\
0 & 1
\end{array}\right]+\left[\begin{array}{ll}
D & A \\
C & B
\end{array}\right] \Delta y+o(\Delta y)
$$

The requirement that the matrix be dissipative is

$$
\left|\left[\begin{array}{cc}
S & U \\
W & R
\end{array}\right]\left[\begin{array}{c}
Z_{1} \\
Z_{2}
\end{array}\right]\right|^{2} \leqq\left|\left[\begin{array}{l}
Z_{1} \\
Z_{2}
\end{array}\right]\right|^{2}
$$

for all complex $n$ vectors $Z_{1}$ and $Z_{2}$. By (24) the requirement holds for $\Delta y \rightarrow 0$ if and only if (5) holds at $y=x$. Conversely, if (5) holds for every choice of $x$ we can subdivide the $x$-interval and use Lemmas 1 and 3 to deduce that the $U R S W$ matrix is dissipative. For this argument it is necessary to have strict inequality in (5), a condition which can be attained by arbitrarily small changes in the coefficients. The statement about unitary matrices can be established by direct computation. Since the method is presented at length in [3], and since a somewhat more general discussion is needed anyway in connection with Theorem XII, we prefer not to belabor the argument here. The result of the analysis is

Theorem III. Let $U, R, S, W$ satisfy (1) for $x \varepsilon I, y \varepsilon I$, where I is an interval of real values, let $R S$ be nonsingular, and let the coefficients $A, B, C, D$ be piecewise continuous. Then the matrix

$$
\left[\begin{array}{ll}
S & U \\
W & R
\end{array}\right]
$$

is dissipative (or unitary, as the case may be) for $x \varepsilon I, y \varepsilon I, y \geqq x$, if and only if the matrix

$$
\left[\begin{array}{ll}
D & A \\
C & B
\end{array}\right]+\left[\begin{array}{ll}
D & A \\
C & B
\end{array}\right]^{*}
$$

is nonpositive (or zero, as the case may be) for $y \varepsilon I$.

As we shall see, this result sharpens REID's Theorem 4.1. It is thought that the present approach makes the structure of the theorem especially clear, and it enables us to prove other theorems of the same type.

Indeed, let $R$ be a relation between pairs of vectors which is transitive in the sense that $\left(Z_{3}, Z_{6}\right) \mathbb{R}\left(Z_{5}, Z_{4}\right)$ and $\left(Z_{1}, Z_{4}\right) \Re\left(Z_{3}, Z_{2}\right)$ implies $\left(Z_{1}, Z_{6}\right) \Re\left(Z_{5}, Z_{2}\right)$. Then if the relation $R$ is ensured by two matrices, it will also be ensured by 
their star product. Hence the question of whether $\mathcal{R}$ is ensured by

$$
\left[\begin{array}{ll}
S & U \\
W & R
\end{array}\right]
$$

will reduce, in general, to the question of whether $R$ is ensured by (24) as $\Delta y \rightarrow 0$. In the foregoing discussion $Q$ is thought to be the relation

$$
\left|Z_{3}\right|^{2}+\left|Z_{6}\right|^{2} \leqq\left|Z_{5}\right|^{2}+\left|Z_{4}\right|^{2} \text {. }
$$

Choice of $R$ as in [3], Theorem VI, enables us to attack the problems of [6].

5. Matrices having property $\odot$. If $X_{i}$ and $K$ are square $n \times n$ matrices, inspection of (2) and (22) yields

$$
\left[\begin{array}{ll}
X_{1} & K \\
X_{2} & X_{3}
\end{array}\right] *\left[\begin{array}{cc}
S & U \\
W & R
\end{array}\right]=\left[\begin{array}{cc}
Y_{1} & U(x, y, K) \\
Y_{2} & Y_{3}
\end{array}\right]
$$

where the $Y_{i}$ are defined by this equation. Because of (25) the star product is an appropriate tool for studying the relation of (7)-(9), and, in particular, for establishing REID's property $\odot$. We shall need the following lemma:

Lemma 5. Let $K$ be a matrix with $\|K\| \leqq 1$. Then there are $n \times n$ matrices $X_{i}$ such that

$$
\left[\begin{array}{ll}
X_{1} & K \\
X_{2} & X_{3}
\end{array}\right]
$$

is unitary.

Let $X_{1}$ be a solution of

$$
X_{1} X_{1}^{*}+K K^{*}=1
$$

as constructed, for example, by diagonalizing $K K^{*}$. Then the first $n$ rows of (26) are mutually orthogonal and have length 1 , so that they form part of an orthonormal basis. Completing the basis by the Gram-Schmidt process, we can fill in the bottom $n$ rows so that (26) is unitary.

Theorem IV. Let $U, R, S, W$ be complex $n \times n$ matrices such that $S$ or $R$ is nonsingular and such that the matrix

$$
\left[\begin{array}{ll}
S & U \\
W & R
\end{array}\right]
$$

is dissipative (i.e., has norm $\leqq 1$ ). Then the matrix

$$
U+S K(1-W K)^{-1} R
$$

is dissipative whenever $K$ is dissipative. If the first matrix is not only dissipative but unitary, then the second is unitary for all unitary $K$. 
With $X_{i}$ given by Lemma 5 we form the product (25). The desired result follows by inspection of

$$
\left[\begin{array}{cc}
Y_{1} & U(x, y, K) \\
Y_{2} & Y_{3}
\end{array}\right]\left[\begin{array}{l}
0 \\
Z_{2}
\end{array}\right]=\left[\begin{array}{c}
U(x, y, K) Z_{2} \\
Y_{3} Z_{2}
\end{array}\right]
$$

if we recall Lemma 3 . It should be noted that Lemma 5 can be satisfied with $X_{3}=0$ in case $K$ is unitary, so that in that case also $Y_{3}=0$.

Since, in general, $Y_{3} \neq 0$ the process yields a sharper inequality than the desired one ( $c f$. the same phenomenon in the proof of [3], Theorem III). To exploit this fact note that $X_{3}^{*} X_{3}=1-K^{*} K$ in Lemma 4. By (25) and (22) $Y_{3}=X_{3}(1-W K)^{-1} R$ so that $Y_{3}^{*} Y_{3}$ can be got without computing $X_{3}$. These considerations yield

Theorem $V$. Let $U, R, S, W$ satisfy (1) for $x \varepsilon I, y \varepsilon I$ where $I$ is an interval of real values, let $R S$ be nonsingular, and let the coefficients be piecewise continuous functions such that

$$
\left[\begin{array}{ll}
D & A \\
B & C
\end{array}\right]+\left[\begin{array}{ll}
D & A \\
B & C
\end{array}\right]^{*} \leqq 0 \text { for } y \varepsilon I .
$$

Let $U(x, y, K)$ be the solution of (1) which equals $K$ at $y=x$, and let $\lambda^{2}$ be the smallest characteristic value for the matrix

$$
R^{*}\left(1-K^{*} W^{*}\right)^{-1}\left(1-K^{*} K\right)(1-W K)^{-1} R .
$$

Then

$$
\|U(x, y, K)\|^{2} \leqq 1-\lambda^{2}
$$

for $x \varepsilon I, y \varepsilon I, y \geqq x$.

Upon replacing $\lambda$ by 0 we get the sufficiency part of ReID's Theorem 4.1.

6. The symmetry of Property P. Since the solutions of (1) satisfy a similar system [1], [2] with some of the variables interchanged, one can surmise that Property $\mathrm{P}$ for $(U R S W)$ implies Property $\mathrm{P}$ for $(W S R U)$. Without reference to the theory of Riccati equations, we shall now show that this is the case.

Theorem VI. Let $U, R, S, W$ and $K$ be complex $n \times n$ matrix with $R$ and $S$ nonsingular. If

$$
\left\|U+S K(1-W K)^{-1} R\right\|<1 \text { whenever }\|K\|<1
$$

then also

$$
\left\|W+R K(1-U K)^{-1} S\right\|<1 \text { whenever }\|K\|<1 .
$$

The author has deduced Theorem VI (as well as Theorems IV and X) by the use of quadratic forms, under a somewhat lighter hypothesis; and the reader 
who prefers a proof along these lines can readily supply one. Discussion here is based instead on the star product, as being more in keeping with the central theme of this paper.

In view of (25), to say that (URSW) (or the corresponding $2 n \times 2 n$ matrix) has Property $\mathrm{P}$ is to say that $U_{1}$ given by

$$
\left[\begin{array}{cc}
- & K \\
- & -
\end{array}\right] *\left(\begin{array}{ll}
S & U \\
W & R
\end{array}\right]=\left[\begin{array}{cc}
- & U_{1} \\
- & -
\end{array}\right]
$$

satisfies $\left\|U_{1}\right\|<1$ wherever $\|K\|<1$; the blank entries are irrelevant. Evidently, if two matrices have Property $\mathrm{P}$, so does their star product, provided the latter is well defined. But this is always the case when $R$ and $S$ are nonsingular, since then we have $\|W\|<1$, as well as the obvious condition $\|U\|<1$ got by setting $K=0$. That is, Property P implies

$$
\left\|S K(1-W K)^{-1} R\right\|<2 \text { for }\|K\|<1
$$

so that, setting $K=K_{1}^{-1}$,

$$
\left\|\left(K_{1}-W\right)^{-1}\right\|<2\left\|R^{-1}\right\|\left\|S^{-1}\right\|
$$

for $\left\|K_{1}\right\|>1$. This cannot hold if $\|W\| \geqq 1$.

The following refinement of Lemma 5 is due to R. Arens:

Lemma 6. In Lemma 5 we can take $X_{3}=-X_{1}$ and $X_{2}=K$.

For proof let the polar decomposition of $K$ be $K=P V$, where $P^{*}=P$ and $V V^{*}=1$. The choice $X_{1}=\left[1-P^{2}\right]^{\frac{1}{2}} V$ yields the desired result.

We now prove Theorem VI. Assuming (27a) form the product

$$
\left[\begin{array}{ll}
S & U \\
W & R
\end{array}\right] *\left(\begin{array}{rr}
X & K \\
K & -X
\end{array}\right]=\left[\begin{array}{ll}
S_{1} & U_{1} \\
W_{1} & R_{1}
\end{array}\right)
$$

where $K$ is fixed with $\|K\|<1$ and $X$ is so chosen that the matrix containing it is unitary. The product

$$
\left[\begin{array}{cc}
0 & K_{1} \\
0 & 0
\end{array}\right] *\left(\begin{array}{ll}
S & U \\
W & R
\end{array}\right] *\left(\begin{array}{cc}
X & K \\
K & -X
\end{array}\right)
$$

can be computed in two ways by the associative law provided, as we now agree,

$$
\left\|K_{1}\right\|<\min \left(1,\left\|W_{1}\right\|^{-1}\right) .
$$

We conclude that

$$
\left\|U_{1}+S_{1} K_{1}\left(1-W_{1} K_{1}\right)^{-1} R_{1}\right\|<1
$$

when (29) holds. But, since (22) shows $R_{1}$ and $S_{1}$ to be nonsingular, (30) can hold for all $K$ in (29) only if $1<\left\|W_{1}\right\|^{-1}$. (This follows as in the discussion of (28).) By (22) the condition $\left\|W_{1}\right\|<1$ is precisely the desired inequality (27b). 
7. A criterion for Property P. If $(U R S W)$ has Property $\mathrm{P}$ it need not be the case that

$$
\left\|\left[\begin{array}{ll}
S & U \\
W & R
\end{array}\right]\right\| \leqq 1
$$

The following criterion shows the extent to which the latter condition is stronger than the former:

Theorem VII. Let $U, R, S$ and $W$ be complex $n \times n$ matrices with $\|U\|<1$ and $\|W\|<1$, and such that the equations

$$
\begin{aligned}
& U_{0}^{*}=U+S W_{0}\left(1-W W_{0}\right)^{-1} R, \\
& W_{0}^{*}=W+R U_{0}\left(1-U U_{0}\right)^{-1} S
\end{aligned}
$$

have a solution with $\left\|U_{0}\right\|<1$ and $\left\|W_{0}\right\|<1$. Let $X$ and $Y$ be so chosen that the matrices

$$
\left[\begin{array}{cc}
X & U_{0} \\
U_{0} & -X
\end{array}\right] \text { and }\left[\begin{array}{cc}
Y & W_{0} \\
W_{0} & -Y
\end{array}\right]
$$

are unitary, and define

$$
\begin{aligned}
& S_{1}=X\left(1-U_{0}^{*} U_{0}\right)^{-1} S\left(1-W_{0} W\right)^{-1} Y, \\
& R_{1}=Y\left(1-W W_{0}\right)^{-1} R\left(1-U_{0} U_{0}^{*}\right)^{-1} X .
\end{aligned}
$$

Then the condition $\left\|R_{1}\right\|\left\|S_{1}\right\| \leqq 1$ implies that (URSW) has Property $\mathrm{P}$, whereas the condition $\left\|R_{1}\right\| \leqq 1,\left\|S_{1}\right\| \leqq 1$ implies (31).

Equations (32) are equivalent to

$$
\left(\begin{array}{cc}
0 & W_{0} \\
U_{0} & 0
\end{array}\right)^{*}=\left(\begin{array}{ll}
S & U \\
W & R
\end{array}\right) *\left(\begin{array}{cc}
0 & W_{0} \\
U_{0} & 0
\end{array}\right) *\left(\begin{array}{ll}
S & U \\
W & R
\end{array}\right)
$$

To prove Theorem VII we shall need the following lemma, which results, by a brief computation, from (22):

Lemma 7. Let $U, R, S, W$ and $X$ be complex $n \times n$ matrices with $\|U\|<1$. If $X$ is so chosen that the matrix containing it is unitary, then

$$
\left(\begin{array}{ll}
S & U \\
W & R
\end{array}\right) *\left(\begin{array}{cc}
X & U^{*} \\
U^{*} & -X
\end{array}\right)=\left(\begin{array}{cc}
S_{1} & 0 \\
W_{1} & R_{1}
\end{array}\right)
$$

for suitable $n \times n$ matrices $S_{1}, W_{1}$ and $R_{1}$.

To prove Theorem VII consider the product

$$
\left(\begin{array}{cc}
Y & W_{0} \\
W_{0} & -Y
\end{array}\right) *\left(\begin{array}{ll}
S & U \\
W & R
\end{array}\right) *\left(\begin{array}{cc}
X & U_{0} \\
U_{0} & -X
\end{array}\right)=\left(\begin{array}{cc}
S_{1} & 0 \\
0 & R_{1}
\end{array}\right)
$$

which can be evaluated by the associative law. Thus, to see that $U_{1}=0$, it is 
best to multiply the two left hand factors, then use (32), and Lemma 7, and finally multiply by the third factor. That $W_{1}=0$ follows by symmetry. One order of computation yields (33), the other order yields the alternative expressions

$$
\begin{aligned}
& S_{1}=X\left(1-U U_{0}\right)^{-1} S\left(1-W_{0} W_{0}^{*}\right)^{-1} Y, \\
& R_{1}=Y\left(1-W_{0}^{*} W_{0}\right)^{-1} R\left(1-U_{0} U\right)^{-1} X .
\end{aligned}
$$

The function $f(K)$ associated with $\left(0 R_{1} S_{1} 0\right)$ is

$$
f(K)=S_{1} K R_{1} .
$$

Evidently $\|f(K)\|<1$ for all $\|K\|<1$ if and only if $\left\|R_{1}\right\|\left\|S_{1}\right\| \leqq 1$, whereas

$$
\left\|\left[\begin{array}{cc}
S_{1} & 0 \\
0 & R_{1}
\end{array}\right]\right\| \leqq 1
$$

if and only if $\left\|S_{1}\right\| \leqq 1$ and $\left\|R_{1}\right\| \leqq 1$.

Now, the defining relations (21) show that the inverse under $*$ multiplication is the same as that under matrix multiplication. In particular,

$$
\left[\begin{array}{cc}
X & U_{0} \\
U_{0} & -X
\end{array}\right]^{-1}=\left[\begin{array}{cc}
X^{*} & U_{0}^{*} \\
U_{0}^{*} & -X^{*}
\end{array}\right]
$$

(as can also be verified by inspection). Since a similar result applies to the matrix containing $Y$, we see by Theorem IV that both inverses have Property P. Solving (34) for the URSW matrix yields Theorem VII.

The interest of Theorem VII is that it admits a converse if, instead of Property $\mathrm{P}$, we consider the property (say $\mathrm{P}^{\prime}$ ) defined by

$$
\left\{\begin{array}{l}
\left\|U+S K(1-W K)^{-1} R\right\|<1 \text { for }\|K\| \leqq 1, \\
\left\|W+R K(1-U K)^{-1} S\right\|<1 \text { for }\|K\| \leqq 1 .
\end{array}\right.
$$

Associated with this condition is the condition

$$
\left\|\left[\begin{array}{ll}
S & U \\
W & R
\end{array}\right]\right\|<1
$$

analogous to (31).

Theorem VIII. Let $U, R, S$ and $W$ be complex $n \times n$ matrices with $\|U\|<1$ and $\|W\|<1$. If (32) has a solution with $\left\|U_{0}\right\|<1,\left\|W_{\mathrm{o}}\right\|<1$, and $\left\|R_{1}\right\|\left\|S_{1}\right\|<1$, then (36) holds, whereas if, in addition, $\left\|R_{1}\right\|<1$ and $\left\|S_{1}\right\|<1$ then (37) holds.

Conversely, if (36) holds then (32) has a solution with $\left\|U_{0}\right\|<1,\left\|W_{0}\right\|<1$ and $\left\|R_{1}\right\|\left\|S_{1}\right\|<1$, whereas if (37) holds then the solution also satisfies $\left\|R_{1}\right\|<1$ and $\left\|S_{1}\right\|<1$.

It suffices to prove the converse, since the direct part follows as in Theorem VII. If (36) is true, the closed convex set of matrix-pairs defined by $\max \left(\left\|U_{0}\right\|\right.$, 
$\left.\left\|W_{0}\right\|\right) \leqq 1$ is mapped into itself by the transformation

$$
\begin{aligned}
& \xi=\left[U+S W_{0}\left(1-W W_{0}\right)^{-1} R\right]^{*}, \\
& \eta=\left[W+R U_{0}\left(1-U U_{0}\right)^{-1} S\right]^{*} .
\end{aligned}
$$

Since the transformation has a fixed point,* (32) has a solution with $\left\|U_{0}\right\| \leqq 1$ and $\left\|W_{0}\right\| \leqq 1$; but actually strict inequality holds, because of Property $\mathrm{P}^{\prime}$. The theorem now follows by inspection of (34).

8. Deductions from the criterion. Any condition on $U, R, S$ and $W$ that ensures $\left\|R_{1}\right\|=\left\|S_{1}\right\|$ in (33) or (35) enables us to deduce (37) from (36). As an illustration we shall prove

Theorem IX. Let $U, R, S$ and $W$ be complex $n \times n$ matrices such that $U^{\prime}=U$, $R^{\prime}=S, W^{\prime}=W$, and $R$ is nonsingular. If (URSW) has Property $\mathrm{P}$ then (31) holds.

Here, as elsewhere, the prime denotes the transpose, not the adjoint. The proof depends on the following lemma, which is due to Robert Steinberg:

Lemma 8. Let $U$ be a symmetric (not necessarily self-adjoint) complex matrix with $\|U\|<1$. Then there is a symmetric matrix $X$ such that

is unitary.

$$
\left[\begin{array}{cc}
X & U \\
U & -X
\end{array}\right]
$$

If the polar decomposition is $U=V P$ it is easily seen that another decomposition is given by $U=U^{\prime}=P^{\prime} V^{\prime}$. Comparing the decompositions $P^{\prime} V^{\prime}=$ $\left(V P V^{-1}\right) V$ we deduce $V P V^{-1}=P^{\prime}$ and $V^{\prime}=V$. It follows that $V P^{n}$ is symmetric for each integer $n \geqq 0$, and hence that $X=V\left(1-P^{2}\right)^{\frac{1}{2}}$ is symmetric also.

To prove the corollary, note first that (WSRU) has Property $\mathrm{P}$ by Theorem VI, so that $(\theta U \theta R \theta S \theta W)$ has Property $\mathrm{P}^{\prime}$ for $0<\theta<1$. Since the inequality

$$
\left\|\left(\begin{array}{ll}
S \theta & U \theta \\
W \theta & R \theta
\end{array}\right)\right\| \leqq 1
$$

implies (31) when we let $\theta \rightarrow 1$, it will suffice to prove the corollary under the assumption that Property $\mathrm{P}^{\prime}$ holds.

The hypothesis yields $\xi^{\prime}=\xi$ and $\eta^{\prime}=\eta$ in (38) from $U_{0}^{\prime}=U_{0}$ and $W_{0}^{\prime}=W_{0}$, and hence we can consider symmetric matrices only in applying the fixed point theorem. Thus $U_{0}^{\prime}=U_{0}, W_{0}^{\prime}=W_{0}$ and by Steinberg's lemma $X^{\prime}=X$ and $Y^{\prime}=Y$. It follows that $R_{1}^{\prime}=S_{1}$, so that $\left\|R_{1}\right\|=\left\|S_{1}\right\| ;$ and this completes the proof.

* This observation is due to I. KAPLANSKY. The author's proof of the existence of a solution was incorrect. 
As another consequence of Theorem VIII we have

Theorem $X$. Let $U, R, S$, and $W$ be complex $n \times n$ matrices with $R$ and $S$ nonsingular. Then (URSW) has Property $\mathrm{P}$ if and only if there is a positive scalar $\lambda$ such that

$$
\left\|\left[\begin{array}{cc}
\lambda S & U \\
W & \lambda^{-1} R
\end{array}\right]\right\| \leqq 1 .
$$

By Theorem IV the condition (39) yields Property $\mathrm{P}$, since $\lambda$ commutes with every matrix. For the converse, suppose first that Property $\mathrm{P}^{\prime}$ holds. Then Theorem VIII gives $\left\|R_{1}\right\|\left\|S_{1}\right\|<1$, so that

$$
\lambda\left\|S_{1}\right\|<1 \text { and } \lambda^{-1}\left\|R_{1}\right\|<1,
$$

provided the positive scalar $\lambda$ satisfies

$$
\left\|R_{1}\right\|<\lambda<\left\|S_{1}\right\|^{-1} \text {. }
$$

Since $U_{0}, W_{0}, X$ and $Y$ are independent of $\lambda$, the matrix

$$
\left[\begin{array}{cc}
\lambda S & U \\
W & \lambda^{-1} R
\end{array}\right]
$$

is transformed by (34) into the matrix

$$
\left[\begin{array}{cc}
\lambda S_{1} & 0 \\
0 & \lambda^{-1} R_{1}
\end{array}\right] .
$$

Since the latter is dissipative by (40), the former must be dissipative too; and that is the desired inequality.

Applying this result to $(\theta U, \theta R, \theta S, \theta W)$ for $0<\theta<1$ we see that there is a $\lambda=\lambda(\theta)$ such that

$$
\left\|\left[\begin{array}{cc}
\lambda \theta S & \theta U \\
\theta W & \lambda^{-1} \theta R
\end{array}\right]\right\|<1
$$

whenever $(U R S W)$ has Property P. Evidently $R \neq 0$ and $S \neq 0$ implies

$$
\theta\|R\| \leqq \lambda(\theta) \leqq \theta^{-1}\|S\|^{-1} \text {. }
$$

Hence we can pick a sequence $\theta=\theta_{n} \rightarrow 1$ such that the corresponding sequence $\lambda\left(\theta_{n}\right) \rightarrow \lambda$, a finite positive limit. This $\lambda$ satisfies (39).

9. Property U. When $R$ and $S$ are nonsingular, (URSW) has Property $\mathrm{P}$, provided

$$
U+S K(1-W K)^{-1} R
$$

is dissipative for all dissipative $K$. If, in addition, this matrix is unitary for all unitary $K$, we shall say that (URSW) has Property U. 
A criterion for Property $U$ can be obtained by noting that the bound (41) is precise whereas (42), in general, is not. Hence the matrices $R_{1}(\theta)$ and $S_{1}(\theta)$ associated with $(\theta U, \theta R, \theta S, \theta W)$ satisfy $\theta\|R\| \leqq\left\|R_{1}(\theta)\right\|<\left\|S_{1}(\theta)\right\|^{-1} \leqq$ $\theta^{-1}\|S\|^{-1}$. Since the space of $n \times n$ matrices is compact we can let $\theta \rightarrow 1$ in such a way that $R_{1}(\theta) \rightarrow R_{1}, S_{1}(\theta) \rightarrow S_{1}$. The matrices $R_{1}$ and $S_{1}$ yield a criterion for Property $\mathrm{P}$ similar to Theorem VIII. If not only Property $\mathrm{P}$ but Property $\mathrm{U}$ holds, the upper and lower bounds of $R_{1}(\theta) S_{1}(\theta)$ can be made as close to unity as desired, by taking $\theta$ close to 1 ; and we deduce, finally, that the matrix (39) is unitary. The converse follows from Theorem IV. These considerations suggest

Theorem XI. Let $U, R, S$ and $W$ be complex $n \times n$ matrices with $R S$ nonsingular. Then (URSW) has Property $\mathrm{U}$ if and only if there is a positive scalar $\lambda$ such that

$$
\left[\begin{array}{cc}
\lambda S & U \\
W & \lambda^{-1} R
\end{array}\right)
$$

is unitary.

We shall give a simple direct proof, instead of filling in the details of the foregoing argument. Let $X$ be so chosen that the matrix containing it is unitary, and consider the product

$$
\left[\begin{array}{cc}
X & W^{*} \\
W^{*} & -X
\end{array}\right] *\left[\begin{array}{ll}
S & U \\
W & R
\end{array}\right]=\left[\begin{array}{cc}
S_{1} & U_{1} \\
0 & R_{1}
\end{array}\right]
$$

(cf. Lemma 7). By Theorem IV, Property U holds for $\left(U_{1} R_{1} S_{1} 0\right)$ whenever it holds for $(U R S W)$. If we can prove Theorem $\mathrm{XI}$ in the special case $W=0$, we can deduce that

$$
\left[\begin{array}{cc}
\lambda S_{1} & U_{1} \\
0 & \lambda^{-1} R_{1}
\end{array}\right]
$$

is unitary for some positive scalar $\lambda$. The identity

$$
\left[\begin{array}{cc}
\lambda S & U \\
W & \lambda^{-1} R
\end{array}\right]=\left[\begin{array}{cc}
X^{*} & W \\
W & -X^{*}
\end{array}\right] *\left[\begin{array}{cc}
\lambda S_{1} & U_{1} \\
0 & \lambda^{-1} R_{1}
\end{array}\right]
$$

now yields Theorem XI in the general case.

Supposing, then, that $W=0$, we have

$$
(U+S K R)\left(U^{*}+R^{*} K^{*} S^{*}\right)=1
$$

whenever $K K^{*}=1$. The choice $K= \pm 1, \pm i$ yields $R U^{*}=U R^{*}=0$ and the equation then simplifies to $U U^{*}+S K R R^{*} K^{*} S^{*}=1$. The choices $K=1$, $K=K$ yield $S R R^{*} S^{*}=S K R R^{*} K^{*} S^{*}$ so that $R R^{*}$ commutes with every unitary $K$. Then $R R^{*}=\lambda^{2}$ where $\lambda$ is a scalar; $\lambda>0$ since $R \neq 0$. The relations which we have obtained so far show that 


$$
\left[\begin{array}{cc}
\lambda S & U \\
0 & \lambda^{-1} R
\end{array}\right]\left[\begin{array}{cc}
\lambda S & U \\
0 & \lambda^{-1} R
\end{array}\right]^{*}=1 ;
$$

and that is the desired result.

Theorem XI yields the following corollaries:

Corollary 1. Let $U, R, S$ and $W$ be complex $n \times n$ matrices with $R S$ nonsingular. If (URSW) has Property $\mathrm{U}$, then so does (WSRU).

Corollary 2. Let $U, R, S$ and $W$ be complex $n \times n$ matrices with $R S$ nonsingular. If (URSW) has Property $\mathrm{U}$ then the set of all unitary matrices $K$ is mapped onto the set of all unitary matrices $K_{1}$ by the transformation

$$
K_{1}=U+S K(1-W K)^{-1} R .
$$

10. Solution of a problem of Reid. In [1] REID raises the interesting question: What is a necessary and sufficient condition for solutions of (1) to have Property $\mathcal{P}$ ? One can also ask the corresponding question for Property $\mathfrak{u}$, where Property $\mathcal{u}$ means that Property $\mathrm{U}$ holds for $x \varepsilon I, y \varepsilon I, y>x$. These questions are answered by the following theorem:

Theorem XII. Let $U, R, S, W$ satisfy (1) for $x \boldsymbol{\varepsilon} I, y \varepsilon I$, where $I$ is an interval of real values, let $R S$ be nonsingular, and let the coefficients $A, B, C, D$ be piecewise continuous. Then (URSW) has Property $\odot$ if and only if there is a real $n \times n$ scalar matrix $\rho(y)$ such that

$$
\left(\begin{array}{ll}
D & A \\
C & B
\end{array}\right)+\left(\begin{array}{ll}
D & A \\
C & B
\end{array}\right)^{*} \leqq\left(\begin{array}{rr}
\rho & 0 \\
0 & -\rho
\end{array}\right) .
$$

Moreover, (URSW) has Property $\mathcal{u}$ if and only if equality holds with $\rho=0$ in (43).

By analogy with Theorems $\mathrm{X}-\mathrm{XI}$ one would expect Property $u$ to follow from equality in (43) with no special restriction on $\rho$. The fact that (1) actually entails $\rho=0$ is reminiscent of Theorem II.

According to Theorem $\mathrm{X}$ Property $\mathrm{P}$ is equivalent to

$$
\left(\begin{array}{cc}
S & U \\
W & R
\end{array}\right]\left[\begin{array}{cc}
\lambda & 0 \\
0 & \lambda^{-1}
\end{array}\right]\left[\begin{array}{ll}
S & U \\
W & R
\end{array}\right]^{*} \leqq\left[\begin{array}{cc}
\lambda^{-1} & 0 \\
0 & \lambda
\end{array}\right] .
$$

If we regard $x$ as fixed and set $y-x=\Delta y$, the initial conditions in (1) applied to (39) show that

$$
\lambda=1+o(1) \text { as } \quad \Delta y \rightarrow 0 .
$$

Hence substitution of (24) into (44) gives

$$
\left[\begin{array}{cc}
\lambda & 0 \\
0 & \lambda^{-1}
\end{array}\right]+\left[\left(\begin{array}{cc}
D & A \\
C & B
\end{array}\right]+\left[\begin{array}{cc}
D & A \\
C & B
\end{array}\right)^{*}\right] \Delta y+o(\Delta y) \leqq\left(\begin{array}{cc}
\lambda^{-1} & 0 \\
0 & \lambda
\end{array}\right] .
$$


If $\rho_{0}=\rho_{0}(x, \Delta y)$ is defined by

$$
\lambda^{-1}-\lambda=\rho_{0} \Delta y
$$

the foregoing equation becomes

$$
\left[\begin{array}{ll}
D & A \\
C & B
\end{array}\right]+\left[\begin{array}{ll}
D & A \\
C & B
\end{array}\right]^{*} \leqq\left[\begin{array}{cc}
\rho_{0} & 0 \\
0 & -\rho_{0}
\end{array}\right]+o(1)
$$

when divided by $\Delta y>0$. It is clear, from this, that $-\rho_{0}$ is bounded below as $\Delta y \rightarrow 0$. If we define $\rho$ (for example) by

$$
\rho=\limsup _{\Delta y \rightarrow 0} \rho_{0}
$$

then (43) holds at the arbitrary point $x$.

For the converse we can suppose strict inequality in (43). That is, let the coefficient matrix be replaced by

$$
\left(\begin{array}{ll}
D & A \\
C & B
\end{array}\right)-\epsilon\left(\begin{array}{ll}
1 & 0 \\
0 & 1
\end{array}\right)
$$

where $\epsilon$ is small but positive. Then the theorem under the hypothesis of strict inequality would give

$$
\left\|U_{\epsilon}+S_{\epsilon} K\left(1-W_{\epsilon} K\right)^{-1} R_{\epsilon}\right\|<1 \text { when }\|K\|<1
$$

for the corresponding solutions. Letting $\epsilon \rightarrow 0$ yields only

$$
\left\|U+S K(1-W K)^{-1} R\right\| \leqq 1 \text { when }\|K\|<1 .
$$

But, since $R$ and $S$ are nonsingular, it is not difficult to show that strict inequality holds. (For example, one can proceed as in the proof of Theorem XI and reduce the discussion to the trivial case $W=0$.) Thus (43) follows.

Assuming, then, that strict inequality holds in (43), we have

$$
\left[\begin{array}{ll}
D & A \\
C & B
\end{array}\right]+\left[\begin{array}{ll}
D & A \\
C & B
\end{array}\right]^{*} \leqq\left[\begin{array}{cc}
\rho & 0 \\
0 & -\rho
\end{array}\right]-\epsilon\left[\begin{array}{ll}
1 & 0 \\
0 & 1
\end{array}\right]
$$

where $\epsilon=\epsilon(x)>0$ at each $x$. The definition of $\lambda$ by

$$
\lambda^{-1}-\lambda=\rho(x) \Delta y
$$

entails $\lambda=1+o(1)$ as $\Delta y \rightarrow 0$. Hence reversal of the foregoing steps yields

$$
\left\|\left[\begin{array}{cc}
\lambda S & U \\
W & \lambda^{-1} R
\end{array}\right]\right\| \leqq 1-\epsilon \Delta y+o(\Delta y) .
$$

This shows that each point $x \varepsilon I$ is in an open interval throughout which $(U R S W)$ has Property P. By the Heine-Borel theorem we can cover any closed subinterval of $I$ by a finite set of such intervals. Since Property $\mathrm{P}$ is preserved by the star product, repeated use of Lemma 1 gives the desired result. 
To discuss Property $u$ let us write, for short,

$$
V=U(x, y, K) \text {. }
$$

If $V V^{*}=1$ whenever $K K^{*}=1$, then $V V_{y}^{*}+V_{y} V^{*}=0$ for these choices of $K$. Since $V$ satisfies (1a) the latter condition reduces to

$$
\left(A V^{*}+D+V B V^{*}+V C\right)+\left(A V^{*}+D+V B V^{*}+V C\right)^{*}=0
$$

when we recall $V V^{*}=1$. Now, by Corollary 2 of Theorem XI, the matrix $V$ runs through all the unitary matrices when $K$ does so. The choices $V= \pm 1$, $V= \pm i$ yield the desired conclusion.

On the other hand, if (43) holds as an equality with $\rho=0$, the matrix $Y=$ $1-V V^{*}$ is found to satisfy

$$
-\frac{\partial Y}{\partial y}=\left(V A^{*}+D\right) Y+Y\left(A V^{*}+D\right) \equiv P Y+Y Q
$$

where $P$ and $Q$ are bounded, since $V_{y}$ exists. Since $V=K$ when $y=x$, we get $Y=0$ at $y=x$ whenever $K$ is unitary. This gives $Y \equiv 0$ by uniqueness, so that Property $u$ holds; and Theorem XII is established.

Given Property U, the scalar matrix $\lambda$ in Theorem XI satisfies

$$
\lambda^{2}=\left(1-U U^{*}\right)\left(S S^{*}\right)^{-1}
$$

for $S$ nonsingular. When the matrix (43) is 0 a straightforward calculation yields $\left(\lambda^{2}\right)_{y}=0$, and the fact that $\lambda=1$ at $y=x$ gives $\lambda \equiv 1$. This remark completes the proof of Theorem III, and establishes the following:

Corollary. Under the hypothesis of Theorem XII, (URSW) has Property $u$ if and only if

is unitary for $x \varepsilon I, y \varepsilon I$.

$$
\left(\begin{array}{ll}
S & U \\
W & R
\end{array}\right)
$$

\section{BIBLIOGRAPHY}

1. W. T. ReId, Solutions of a Riccati Matrix Differential Equation as Functions of the Initial Values, J. Math. and Mech. 8 (1959), pp. 221-230.

2. R. M. RedhefFer, On Solutions of Riccati's Equation as Functions of the Initial Values, JRMA 5 (1956), pp. 835-848.

3. R. M. Redheffer, The Riccati Equation: Initial Values and Inequalities, Math Annalen 133 (1957), pp. 235-250.

4. R. M. Redheffer, Remarks on the Basis of Network Theory, J. Math. and Physics 28 (1950), pp. 237-258.

5. J. Mycielski \& S. Paszkowski, Sur un problème du calcul de probabilité I, Studia Math. 15 (1956), pp. 188-200.

6. S. Paszkowski, Sur un problème du calcul de probabilité II, Studia Math. 15 (1956), pp. 274-299. 